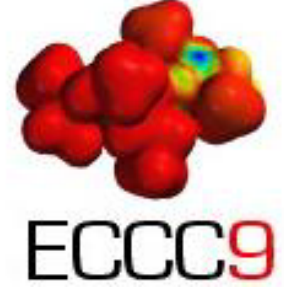

\title{
The Interpretation of the Short Range Disorder in the Fluorene- TCNE Crystal Structure
}

\author{
Gianluca Croce ${ }^{1}$, Aldo Arrais ${ }^{1}$, Eliano Diana ${ }^{2}$, Bartolomeo Civalleri ${ }^{2}$, Davide Viterbo ${ }^{1}$, Marco \\ Milanesio $^{1^{*}}$ \\ ${ }^{1}$ Dipartimento di Scienze e Tecnologie Avanzate, Università del Piemonte Orientale, Corso Borsalino \\ 54, I-15100 Alessandria, Italy \\ ${ }^{2}$ Dipartimento di Chimica I.F.M., Università di Torino, Via P. Giuria 7, I-10125, Torino, Italy. \\ Tel.: +39 (0) 131287 414, Fax: +39 (0) 131287 416, E-mail: marco.milanesio@mfn.unipmn.it \\ URL: http://www.mfn.unipmn.it/ marcomi/ \\ *Author to whom correspondence should be addressed.
}

Received: 1 May 2003 / Accepted: 18 July 2003 / Published: 20 February 2004

\begin{abstract}
The interpretation of the short-range static and/or dynamic disorder in a crystal structure from X-ray data is often a rather complex problem. Recently, we synthesized and characterized three co-crystalline complexes between fluorene, showing $C_{2 v}$ symmetry, and three electron withdrawing $D_{2 h}$ compounds, employing FTIR and Raman spectroscopy and single crystal X-ray diffraction techniques. The crystal structures of the complexes of fluorene with the three different electron withdrawing molecules are disordered in the solid state and only approximate structures were obtained by refinement of the single crystal data. Indeed, the fluorene moiety presents a very irregular geometry, showing for example C-C bonds ranging from $1.25 \AA$ to $1.75 \AA$. Graphical inspection of the solved crystal structures indicates that the fluorene molecules in these co-crystalline complexes can assume two possible positions, both with $50 \%$ population. A two-step procedure to improve the disordered models is described. At first, the two possible ordered structures, with the fluorene molecule in only one of the two populated positions, are "separated" by molecular graphic techniques and then their geometry is fully optimized employing the periodic $a b$ initio "CRYSTAL" code to obtain a chemically sensible model with reasonable distances and angles. The main aim of this communication is to demonstrate that ordered models can be obtained, starting from a disordered crystal structure, focusing our attention on the fluorene-TCNE molecular complex.
\end{abstract}

Keywords: disordered crystal structures, molecular crystals, ab initio periodic calculations. 


\section{Introduction}

Short-range static and/or dynamic disorder is an "undesirable guest”, which affects a considerable number of crystal structures. When some disorder is found in a crystal structure, it is often impossible to obtain sensible geometric features such as bond distance and angles, intermolecular contacts and packing arrangements. Theoretical calculations can then be used to achieve a better interpretation of the electron density from a crystal structure presenting various degrees of structural short-range disorder.

Recently, we decided to study the possibility of co-crystallizing the fluorene molecule, which presents a $C_{2 v}$ symmetry, with an electron withdrawing molecular unit of higher symmetry, in order to obtain a charge transfer (CT) complex. We have chosen the fluorene molecule because it is well known for its optical responses and, for these properties, its use is investigated in active optically designed materials [1-3]. We employed, as fluorene counterparts, tetracyanoethylene (TCNE in Figure 1), 1,2,4,5 tetracyanobenzene (TCNB) and 7,7,8,8-tetracyanoquinodimethane (TCNQ), all presenting $D_{2 h}$ symmetry. We were aware that the crystal structures of fluorene containing molecular complexes were disordered [4-7].

The three complexes have been characterized employing spectroscopic techniques (FTIR and Raman spectroscopy) and single crystal X-ray diffraction analysis. The analysis of the X-ray diffraction data was complicated by the difficulty in assigning the correct space group, because of the uncertainty on the presence or not of an inversion center and the possible disorder of the fluorene unit. Therefore some $a b$ initio periodic theoretical calculations have been performed, in order to solve the problem inherent to the interpretation of the crystal data. All the experimental results, together with the synthesis and the crystallization techniques, will be fully described in a separated paper [8]. The aim of this paper is to show that sensible models of the crystal structures can be obtained by a two step procedure: (i) the disordered experimental structure is first separated into its components by graphical manipulation; (ii) then the geometry is optimized by means of $a b$ initio quantum-chemical calculations, carried out with the CRYSTAL code [9]. The Hartree-Fock (HF) level of theory was adopted within the LCAO approximation and periodic boundary conditions. Recently, thanks to the implementation in CRYSTAL [9] of the analytical HF nuclear gradient [10,11], geometry optimization

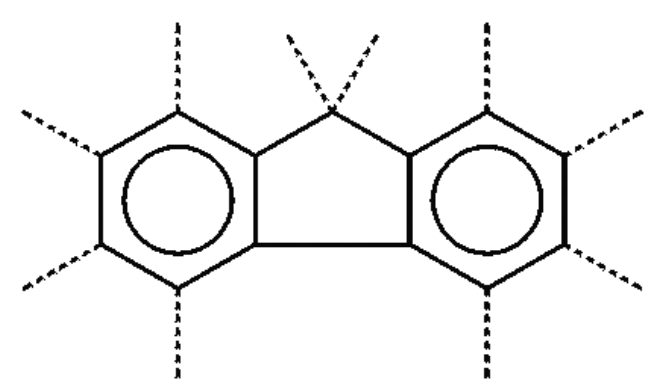

Fluorene

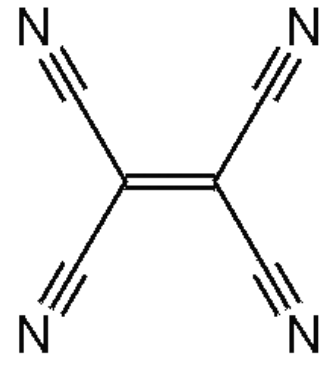

TCNE

Figure 1. Fluorene and its $D_{2 h}$ counterpart in the co-crystalline complex (C-H bonds are indicated by dotted lines). 
of periodic systems has become feasible in routine calculations [12]. Thus crystal structures can be optimized and an $a b$ initio quantum mechanical structure refinement can be performed. In particular, here we report in details the above-described procedure, applied to the structure of the Fluorene/Tetracyanoethylene complex (hereafter named complex 1).

\section{Computational Details}

All calculations have been performed by using the CRYSTAL code [9]. A fixed-cell unconstrained optimization of the atomic coordinates is performed by fixing the cell parameters to the values obtained from the X-ray diffraction experiments. The forces acting on the atoms are obtained by using the recent implementation in CRYSTAL of analytical HF gradients [10], and used to relax the atom positions towards equilibrium using a modified conjugate gradient algorithm as proposed by Schlegel [13]. Standard Pople 3-21G, 6-21G, 6-21G(d) and 6-21G(d,p) basis sets [14] were employed to obtain the optimized geometries and to compute the corresponding lattice energy (L.E.), employing the following relation:

$$
\text { L.E. }=\mathrm{E}(\mathrm{X})_{\mathrm{CRYSTAL}}-\mathrm{E}(\text { Fluorene })_{\text {MOLECULE }}-\mathrm{E}(\mathrm{TCNE})_{\text {MOLECULE }}
$$

where X can be complex $\mathbf{1 a}$ or $\mathbf{1 b}$.

Default tolerances for the integral calculations (except for T5 = 14) [9] and geometry optimization [12] were adopted. The number of reciprocal lattice points (k-points) at which the Hamiltonian matrix has been diagonalized is 36 , corresponding to a shrinking factor $\mathrm{S}=4$ [9].

\section{Results}

\section{From a Disordered Crystal Structure to an Ordered Model}

The crystal structure of complex $\mathbf{1}$ is disordered in the solid state and only an approximate structure can be obtained by refinement of the single crystal data. Fluorene (the electron donating $\mathrm{C}_{2 \mathrm{v}}$ molecule) always presents a very irregular geometry, showing for example $\mathrm{C}-\mathrm{C}$ bonds ranging from $1.25 \AA$ to $1.75 \AA$. Conversely, the electron withdrawing $\mathrm{D}_{2 \mathrm{~h}}$ molecules (i.e. the counter part of fluorene in the complexes) are ordered, thanks to their higher symmetry as already described by Muhle et al. [5]. Graphical inspection of the crystal structures suggests that the fluorene molecules in these cocrystalline complexes can assume two possible positions (indicated by black and white bonds in Figure 2), related by an inversion center, both with $50 \%$ population.

Molecular manipulations by means of the MOLDRAW [15] and XP [16] programs were performed to generate the two possible models with the two ordered positions of the fluorene molecule without modifying the crystallographic periodicity. This artificial graphical separation process is illustrated in Figure 2 for complex $\mathbf{1}$. The model crystal structure with the combination of the fluorene molecule 

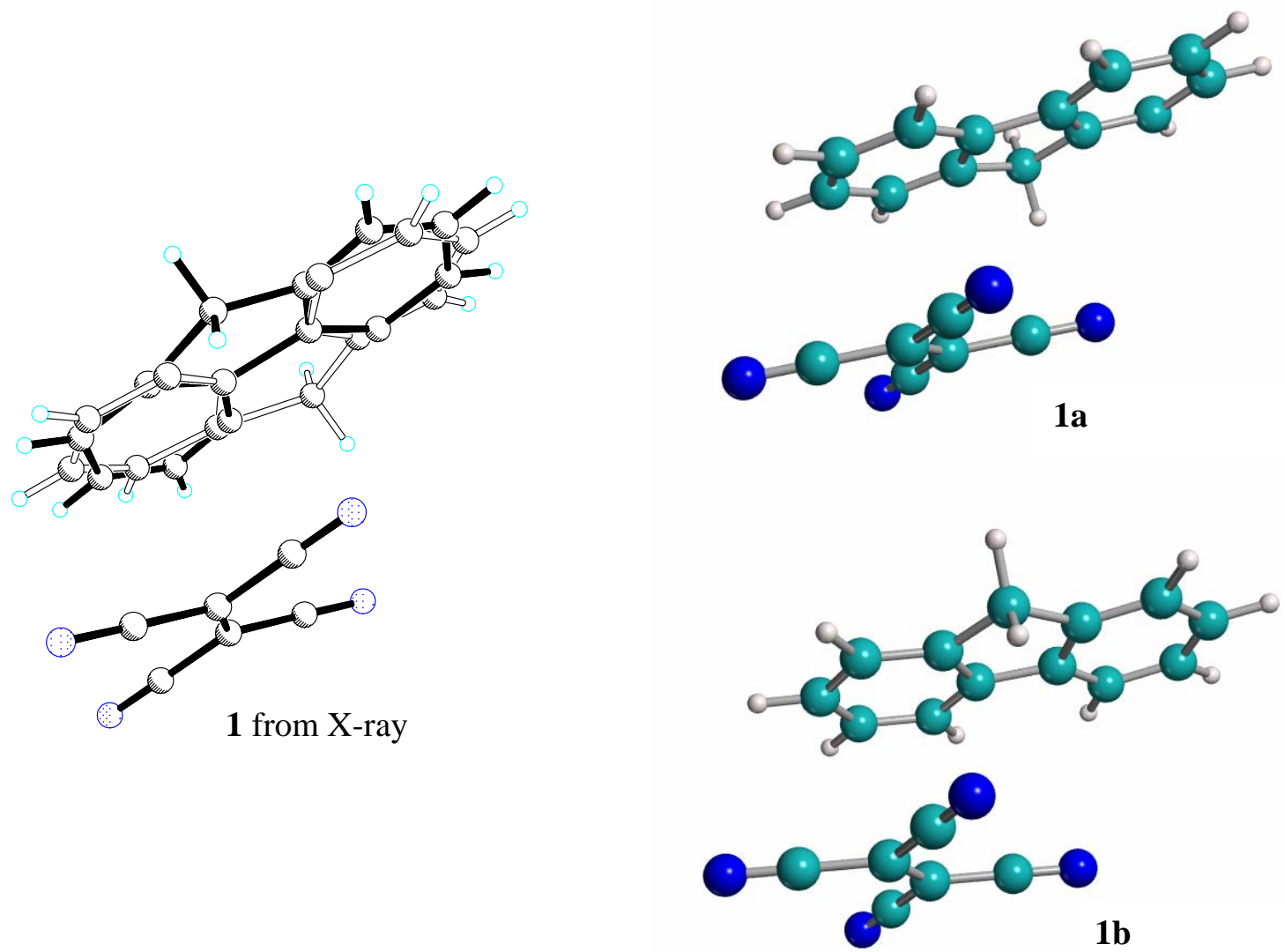

Figure 2. Sketch of the graphical separation process used to obtain the two distinct ordered structures from the disordered crystal structure of $\mathbf{1}$, where the black and white bonds indicate the $\mathbf{1 a}$ and $\mathbf{1 b}$ positions respectively.

labeled a with its TCNE counterpart is named 1a, and that with the combination of molecule $\mathbf{b}$ with its TCNE counterpart is named $\mathbf{1 b}$. Of course, the model ordered structures $\mathbf{1 a}$ and $\mathbf{1 b}$, derived from the centrosymmetric P-1 X-ray structure, belong to the related non-centrosymmetric space group P1. Starting from these model structures their full geometry optimization was performed to obtain sensible geometries and to verify whether the a and $\mathbf{b}$ positions present the same stability. Besides, an estimate of the lattice energy (L.E.) was obtained by subtracting the gas phase energy of the isolated molecules from the crystal energy, according to eq. 1.

\section{Geometry Optimization}

In this paragraph we describe the geometric features of compounds $\mathbf{1 a}$ and $\mathbf{1 b}$ after geometry optimization of the model structures (Figure 2) at the HF level, employing increasingly larger basis sets.

No significant changes in the values of bond distances and angles can be observed passing from the $\mathrm{HF} / 3-21 \mathrm{G}$ to the HF/6-21G(d,p) level of theory and therefore only the results obtained at the HF/6- 
21G(d,p) level are reported.

The more dramatic changes between the X-ray structure and that after geometry optimization involve the 5-member ring of fluorene, shown in Figure 3 with the adopted labeling scheme. Figure 3 and Table 1 show the comparison between the geometrical features of the 5-member ring before and after full geometry optimization of 1a. It can be seen that the geometry optimization gives a chemically sensible model with the expected bond lengths and angles, starting from the rather deformed geometry obtained from the X-ray data. Indeed the optimized bond distances and angles (see Figure 3 and Table 1) are rather similar to those from the X-ray crystal structure of fluorene alone [17], which is not disordered. The comparison between the geometry of fluorene alone in the crystal structure [17] and of fluorene after $\mathrm{HF} / 6-21 G(\mathrm{~d}, \mathrm{p})$ full geometry optimization suggests that this level of theory is sufficiently accurate to describe the geometry of fluorene itself. Therefore the discrepancies between the calculated and experimental geometries of fluorene in complex $\mathbf{1}$ are certainly due to the structural disorder of the X-ray structure of complex 1.

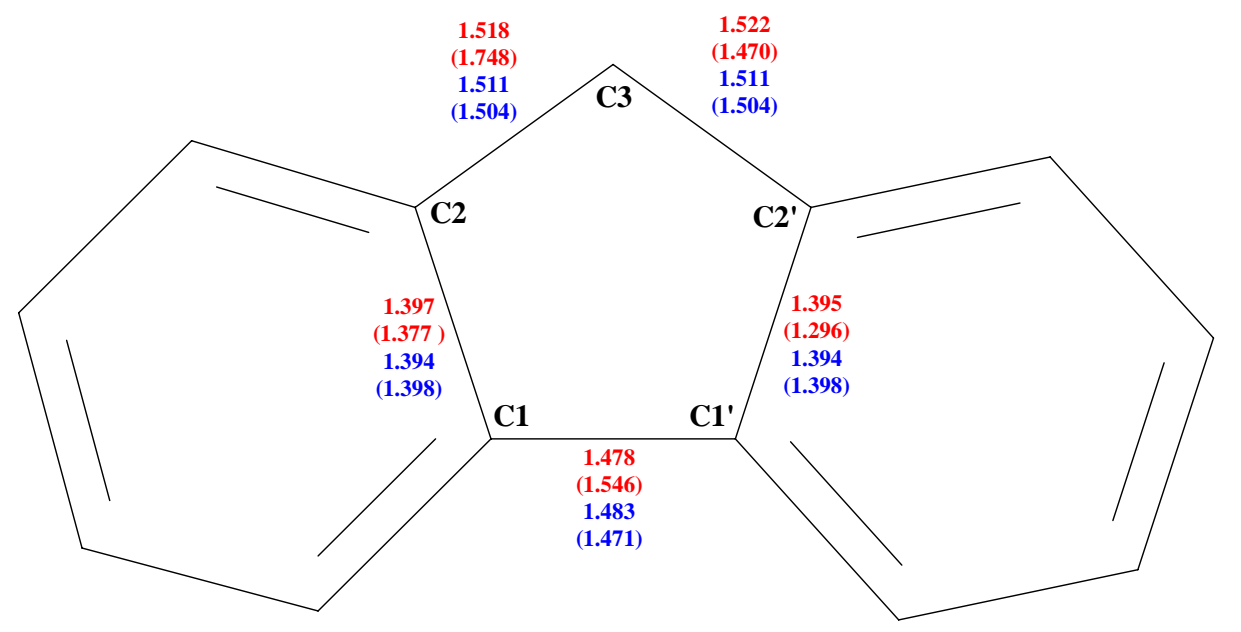

Figure 3. The 5-member ring of fluorene and its labeling. The values after geometry optimization are compared with the corresponding values from the X-ray data refinements (in parenthesis) of 1a (in red) and of fluorene alone [17] (in blue).

Table 1. Bond angles of the 5-member ring of fluorene in 1a fluorene alone [17] from the X-ray data and after full geometry optimisation.

\begin{tabular}{ccccc}
\hline $\begin{array}{c}\text { Angle } \\
{ }^{\circ} \text { ] }\end{array}$ & $\begin{array}{c}\text { 1a } \\
\text { 6-21G(d,p) }\end{array}$ & $\begin{array}{c}\text { 1a } \\
\text { X-ray }\end{array}$ & $\begin{array}{c}\text { Fluorene }^{(\$)} \\
6-21 G(d, p)\end{array}$ & $\begin{array}{c}\text { Fluorene }^{(*)} \\
\text { X-ray }\end{array}$ \\
\hline C1-C2-C3 & 110.3 & 102.4 & 110.4 & 110.4 \\
C2-C3-C2' & 102.2 & 120.0 & 102.5 & 102.7 \\
C3-C2'-C1' & 110.5 & 102.3 & 110.5 & 110.4 \\
C2'-C1'-C1 & 108.4 & 107.2 & 108.3 & 108.3 \\
C1'-C1-C2 & 108.5 & 128.3 & 108.7 & 108.3 \\
\hline
\end{tabular}

(\$) After HF/6-21G(d,p) periodic full geometry optimisation of the geometry from ref [17];

(*) From reference [17]. 
In Figure 4 the crystal packing of the complex 1a, after geometry optimization at the HF/6-21G(d,p) level, is shown. The intermolecular interactions driving the crystal packing are:

- the weak C-H $\cdots \cdot \mathrm{N}$ interactions between molecules on the same plane (dotted lines in Figure 4), with $\mathrm{H} \cdots \cdots \cdot \mathrm{N}$ distances in the range 2.55-2.91 $\AA$;

- the stacking interactions between the fluorene and TCNE molecules on adjacent parallel planes. The distance between the centers of the $\mathrm{C}=\mathrm{C}$ bond in TCNE and of the facing phenyl group in fluorene (the two moieties, involved in the CT, shown in Figure 4), is about 3.31 $\AA$. This value is very close to the inter-planar distance $(\sim 3.28 \AA)$, indicating an almost perfect facing between TCNE and the phenyl group in fluorene.

It is worth noting that the intermolecular distances obtained from the disordered crystal structures were too crude to allow a reliable discussion of the above packing geometry.

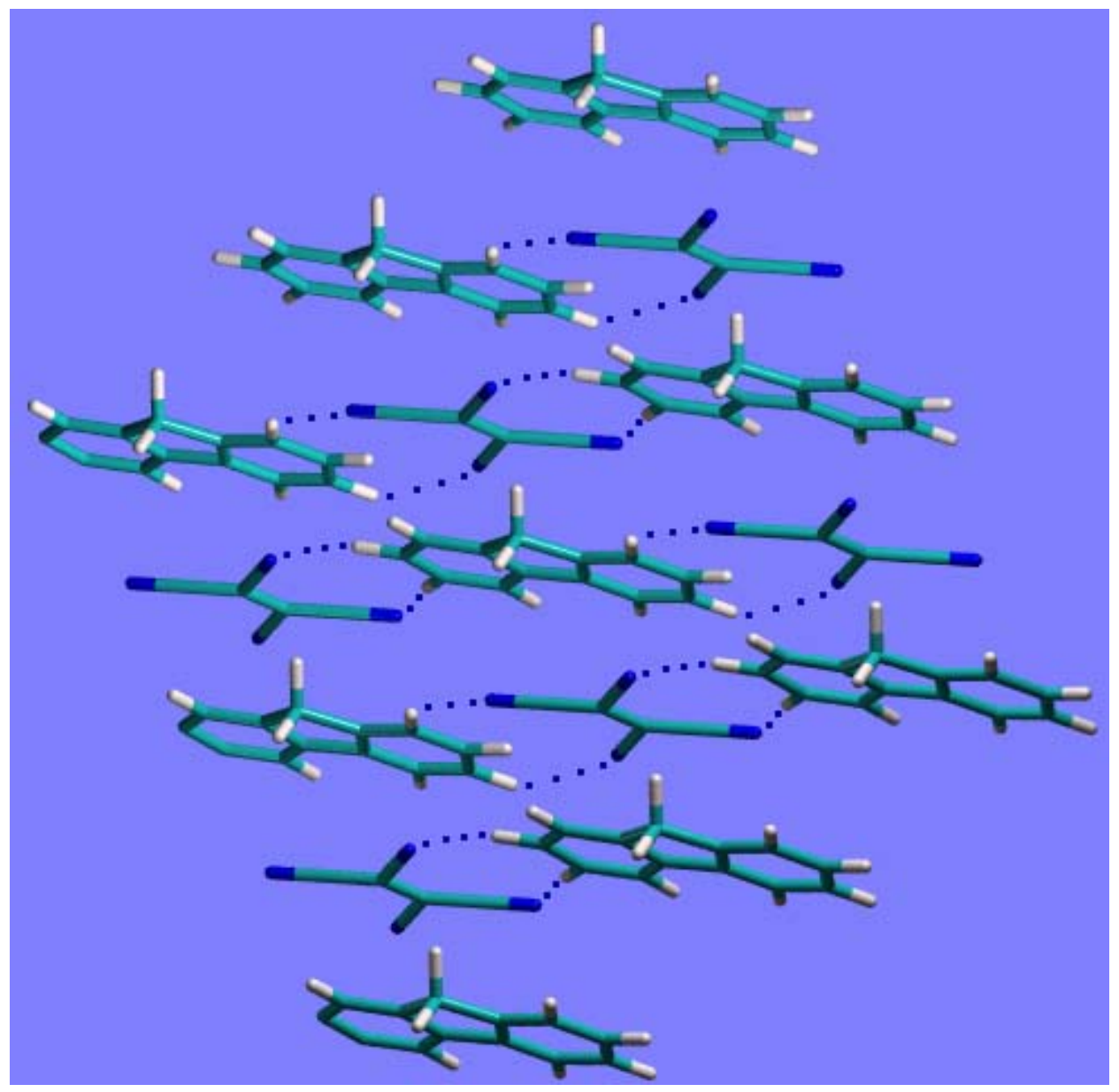

Figure 4. The crystal packing arrangement of compound 1a after geometry optimization. 


\section{Energy Results}

In Table 2 the energetic features of the studied models after full geometry optimization are reported. The optimized structures $\mathbf{1 a}$ and $\mathbf{1 b}$ retain an enantiomeric relation and have very similar total energy values after geometry optimization. Indeed the two fluorene dispositions differ by less than $0.2 \mathrm{~kJ} / \mathrm{mol}$ at the HF/6-21G(d,p) level of theory and therefore can be considered equivalent. The presence of the inversion center (relating the two equivalent fluorene positions in the actual disordered crystal structure) is then explained and confirmed.

As already mentioned, the described procedure also allows an approximate estimate of the lattice energy (see eq. 1). For instance the value calculated for 1 at the HF/3-21G level is: L.E. $=136.2 \mathrm{~kJ} / \mathrm{mol}$. The comparison of the values obtained for different complexes can be correlated with the experimental spectroscopic evidences on the weak interactions holding together the complexes in the solid state and in solution (a detailed comparison will be reported in a separated paper [8]).

Table 2. Total energy for the two model structures of compound $\mathbf{1}$ (data in Hartrees, 1 Hartree = $2625.5 \mathrm{~kJ} / \mathrm{mol}$ ) and relative stability $\Delta \mathrm{E}=\mathrm{E}_{\mathbf{1 a}}-\mathrm{E}_{\mathbf{1 b}}$ (in $\mathrm{kJ} / \mathrm{mol}$ ) at different levels of calculation.

\begin{tabular}{lccc}
\hline & 1a & 1b & $\Delta \mathrm{E}$ \\
\hline HF/3-21G & -937.7946535 & -937.7941936 & -1.21 \\
HF/6-21G & -942.0021077 & -942.0021458 & 0.10 \\
HF/6-21G(d) & -942.4568083 & -942.45694895 & 0.37 \\
HF/6-21G(d,p) & -942.4857835 & -942.4858601 & 0.20 \\
\hline
\end{tabular}

\section{Conclusions}

In this work the CRYSTAL code was employed to elucidate the disordered crystal structure, obtained from X-ray single crystal analysis on a molecular complex of fluorene with the electron withdrawing TCNE molecule (Figure 1). The crystal structure analysis of complex 1 (Figure 2 left) indicated that the fluorene molecule is disordered over two symmetry related positions (1a and $\mathbf{1 b}$ in Figure 2 right) in the centrosymmetric $P-1$ crystal structure. Ab initio periodic calculations on the two graphically separated non-centrosymmetric P1 model crystal structures indicated that these two positions are equivalent from the geometric and the energetic point of view. The presence of an inversion center is thus explained and confirmed and the correctness of the X-ray data refinement in the $P-1$ space group is verified. The geometry of the disordered fluorene obtained in this way is poorly defined and only by the $a b$ initio full geometry optimization of the $P 1$ model structures could a sensible fluorene moiety, with the expected bond distances (Figure 3) and angles (Table 1) and intermolecular contacts (Figure 4), be obtained, together with an estimate of the lattice energy.

The main scope of the present paper is to show that the deformed geometry obtained from the X-ray analysis of a disordered crystal structure can be improved by the use of the new features of the 
CRYSTAL code, which allow the $a b$ initio full geometry optimization of a periodic structure. A full account of the crystallographic and theoretical implications of the described procedure will be given elsewhere. [18].

\section{Acknowledgements}

Helpful suggestions on some computational aspects by Dr. M.o Causà, Dr. R. Orlando (Università del Piemonte Orientale) and Prof. R. Dovesi (Università di Torino) are acknowledged.

\section{References and Notes}

1. Hummel R.L.; Ruedenberg K. J. Phys. Chem., 1962, 66, 2334.

2. Siegel S.; Judeikis H.S. J. Phys. Chem. 1966, 70, 2205.

3. Bree A.; Zwarich R. J. Chem. Phys. 1969, 51, 903.

4. Hertel, R. Z. Phys. Chem. (Leipzig) 1930, B11, 77.

5. Herbstein F.H.; Kaftory M.; Regev H. J. Appl. Crystallogr. 1976, 9, 361.

6. Mariezcurrena R.A.; Russi S.; Mombrú A.W.; Suescun L.; Pardo H.; Tombesi O.L.; Frontiera M.A. Acta Crystallogr., Sect.C 1999, 55, 1170.

7. Muhle W.; Krzystek J.; Schutz J.U.; Wolf H.C.; Stigler R.D.; Stezowski J.J. Chem. Phys. 1986, 108, 1.

8. Arrais A.; Boccaleri E.; Croce G.; Milanesio M.; Orlando R.; Diana E. Cryst. Eng. Comm. 2003, 5, 388.

9. Saunders, V.R.; Dovesi, R.; Roetti, C.; Orlando, R.; Zicovich-Wilson, C.M.; Harrison, N.M.; Doll, K.; Civalleri, B.; Bush, I.J.; D'Arco, P.; Llunell, M. CRYSTAL03 User's Manual, (Universita' di Torino, Torino, 2003).

10. Doll K.; Harrison N.M.; Saunders V.R. Int. J. Quantum Chem. 2000, 82, 1.

11. Doll K. Comp. Phys. Comm. 2001, 137, 74.

12. Civalleri B.; D'Arco Ph.; Orlando R.; Saunders V.R.; Dovesi R. Chem. Phys. Lett. 2001, 348, 131.

13. Schlegel H.B. J. Comp. Chem. 1982, 3, 214.

14. Hehre W.J.; Radom L.; Schleyer, P.v.R.; Pople J.A. Ab initio molecular orbital theory, 1986, Wiley, NY.

15.Ugliengo P.; Viterbo D.; Chiari G. Z. Krist. 1993, 207, 9. (web site: http://www.ch.unito.it/ifm/ fisica/moldraw/moldraw.html).

16. XP in SHELXTL - (C. 1998. Madison Winsconsin, 53719 USA, Bruker AXS, Inc. 1998.

17. Gerkin R.E.; Lundstedt A.P.; Reppart W.J. Acta Crystallogr., Sect.C (Cr.Str.Comm.) 1984, 40, 1892.

18. Milanesio M.; Croce G.; Civalleri B.; Orlando R.; Viterbo D.; Dovesi R. in preparation for submission to Acta Crystallogr., Sect.A.

(C) 2004 by MDPI (http://www.mdpi.net). Reproduction for noncommercial purposes permitted. 